\title{
A Framework of Computational Experiment within Special Artificial Society Scene
}

\author{
Zhang Peng, Fan Zong Chen, Chen Bin, Meng Rong Qing, and Qiu Xiao Gang
}

\begin{abstract}
Recently computational experiment based on special artificial society scene have played an important role to deal with various problems in emergency management. In this paper, firstly we summarize the basic theory of artificial society and emergency management. Second, we provide the method of constructing artificial society scene, including building basic models and generating population database. Then, we propose a large-scale individual-based framework of computational experiment within special artificial society scene, namely KD-ACP. Finally, we give a case of H1N1 influenza to introduce the platform workflow in the emergency management.
\end{abstract}

Index Terms-Artificial society, computational experiment, scene and response, scenario generation.

\section{INTRODUCTION}

Since the outbreak of SARS epidemic in 2003, many academician carry out a series of comprehensive researches about the emergency management [1]. However there are many non-structural or semi-structural problems in social system, which are the obstacle of emergency management. In the study of the social problems, the main issues need to be considered as follows:

1) The social problems are always not isolated, and the inherent mechanism is hard to distinguish.

2) Researchers always face the problem of lacking data, and the accuracy of the data is also doubtful.

3) Different people often have different views on the same issue due to their different experiences and backgrounds.

Artificial society provides a new perspective to analyze the complexity of social system, comparing to the simulation society. Simulation society is a top-down approach, which decomposes the society and analyzes the natural characters of the social systems. While artificial society is a bottom-up approach, which makes use of computer technology and agent technology to foster the growth of virtual society, and real plays the status and development trend of the artificial society.

Constructing artificial society and doing computational experiment is a good approach to study the complex social system and individual behavior in recent years [2]. And the process will involve multiple disciplines knowledge, such as

Manuscript received February 1, 2014; revised April 4, 2014.This work was supported in part by National Nature Science Foundation of China under Grant No. 91024030 and No.91324013.

The authors with the College of Information System and Management, National University of Defense Technology, Changsha, Hunan P. R. China, 410073 (e-mail: zhangpeng_yes@163.com). computer science, sociology, and complexity science [3]. Luckily, it's possible to do large-scale individual-based computational experiments with the increasing computing capability. Recently some researchers also try to introduce the computation experiment to the emergency management [4]. By constructing the initial emergency scenario and loading immediate measures, we can evaluate the efficiency of our action and propose scientific advice to the government for making emergency response plan.

\section{THE ARTIFICIAL SOCIETY THEORY AND RESEARCH METHODS}

\section{A. Agent Based Modeling and Simulation}

Agent-based modeling and simulation offers a way to model social systems composed of agents, which can interact with each other according to some simple rules [5]. Agent-based systems are often self-organize where agents can learn from their experiences, and adapt their behaviors to the environment. In these systems, each agent usually has the following characteristics [6]:

1) Activity: each agent independently acts according to the rules and their own pre-defined behaviors.

2) Autonomy: each agent is free for activity and makes independent decisions.

3) Heterogeneity: each agent may develop individuality according to different environment.

The diversity exists among agents can be observed by modeling agents individual. The macroscopic phenomenon is not just a scale-up replication of the simple behavior of individual. We can model the dynamics complex systems and interpret the macroscopic phenomenon using agent-based system [7]. Agent-based modeling has proved useful for a wide range of problems in physics, biology, economics, social science and geospatial simulations [8]. Some software can support the agent-based modeling and simulation, such as Swarm, Mason, Netlogo and Repast.

\section{B. The ACP Theory}

In 2004, Wang Feiyue proposed the ACP approach, namely artificial societies, computational experiments and parallel execution, which supports to modeling, analyze and control the complex social systems [9]. We can construct artificial societies to simulate the operation of the real world, and foster the artificial society to present a variety of possible future. The real world and artificial society can interact with each other and realize the co-evolution of both systems.

We can do some complex computational experiment based 
on artificial society [10]. First, build the individuals and environment entities; second, allow these agents interact with each other in the artificial environment according to some relatively simple rules; third, collect data to summarize the operation mechanism of artificial society and interpret the macroscopic phenomena. Comparing to common agent-based systems, the artificial society has its typical character as follows.

1) Large-scale: the individual number of artificial society is much more than that of common agent-based system, and the individual usually has more attribute and behaviors.

2) Multi-layer network: the relationships and interactions among individuals are usually complex, and each individual may be in different network at the same time.

\section{Scenario and Response Theory}

Emergencies can also be called unexpected events, which are always company with catastrophic results. They can cause huge economic losses, environmental damage, personal injury, and even endanger the political security of the country [11]. According to the nature and mechanism, emergencies events can be divided into natural disasters, accidents, public health incidents and social safety incidents. Scenario is the situation of emergency, which clearly depicts the major possible threats, and the evolution trend of the event. The geographical parameter, social environments and weather conditions should also be included in the scenario, which is the initial state of the computational experiment.

Emergency response is the core stage of emergency management. The alert time and disposal time is limit because of uncertain factor in the process. All emergency departments and organizations should collaborate to take immediate actions to decrease lose. First, set up an emergency command center and make the appropriate emergency plans. Second, collect the emergency information and report to the public timely. Then, collect all possible strength to support the personnel, technology, medication, materials, equipment, transportation and communications. The Chinese government sticks to the emergency management principles of people orientation, hazard reduction, crisis consciousness and precaution crucial. In the process of building a harmonious society, the government proposes to construct one plan and three systems, namely the basic emergency response plane, comprehensive emergency response plan system, emergency management system, emergency response mechanism and emergency related legal system [12].

\section{CONSTRUCT THE INITIAL ARTIFICIAL SOCIETY SCENARIO}

\section{A. Built Basic Models}

Artificial social system contains a series of basic models, such as individuals, environment entities, and the social network. Fig. 1 shows the relationship of individual, environment and social network in the artificial society. Individual is the basic element, which is always autonomous and self-directed. The environment provides the basic operation parameters of computation experiments, such as location, temperature and humidity. The social network describes the relationship of the individuals. Models must be developed as modules so that they can be assembled, interoperable and reusable.

\section{1) Individual model}

Individual is the most active element in artificial society, which can interact with each other and evolve the artificial society. We must analysis the social characteristics of the individual and design its general structure, including basic attributes and behaviors. The static attributes are usually never changed in simulation, such as id, name and gender. While the dynamic attributes may vary by interaction, such as state and location. Behaviors are often described by simple rules, so we must define some rules to drive the interaction. For large-scale agent based simulation, we design schedule to simulate the action sequences of individuals. Based on single-agent model, we can define types of social structure to simulate the function of a group of individual, such as government.

Besides the basic attributes and behaviors, other modules should be considered in order to meet the different domain simulation. In public health, the health module should be involved, which may include the health state, immunity state, blood type and so on. The module should contain the state transition diagram of individuals. In public security, the mentally factors can affect the behavior of individuals. So the cognitive psychology module is needed, which is usually composed of a set of rules and types of reaction.

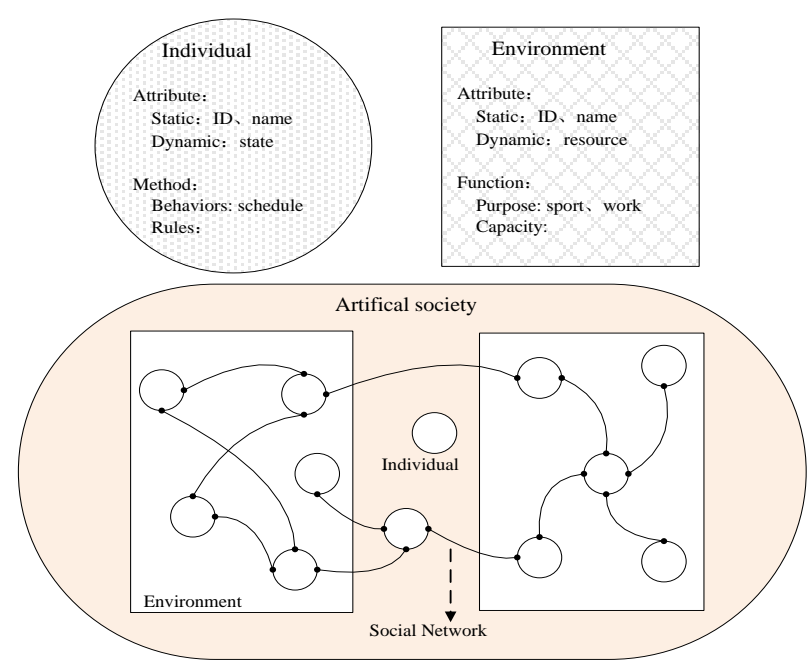

Fig. 1. Individual, environment and social network in the artificial society.

\section{2) Environment model}

Environment is the indispensable part of artificial society. The environment models include the physical environment entities, climate module, traffic module, culture module and so on. Physical environment provide the space where individuals active in, such as school, government and hospital. Usually, Environment entities must contain the geography location (longitude and latitude), function, and capacity.

Based on the historical climate data from meteorological stations, we can define several types of artificial climate. In simulation, all individuals and environment entities can operate in the pre-defined climate. Traffic modules contain the road nets and traffic lines. According to the historical monitoring data, we can simulate the traffic flow at different 
time. Cultural module provides the background of social attitudes, which may affect the behavior patterns of the agent.

\section{3) Multi-social network model}

Society networks depict the relationship of individual, which is the basic condition of the interaction among individual. In artificial society, multiple social networks are involved including family, classmate, neighborhood and coworker. These social networks describe the steady relationships among individuals, which are called strong links. While there are some relationship established temporally in special condition, which are called weak links.

In artificial society, the relationship among individuals is always complex, and the social networks are always multi-layers. For example, an individual may has its classmate network, coworker network, friend network and so on. Individual usually plays different role at the same time, and interact with each other through different social networks.

\section{B. Generate Artificial Database}

For large-scale computational experiments, synthetic population and virtual physical environment are the basis for further individual-based modeling and simulation. There are three manners to establish the artificial database:

1) Make use of the statistical data of each individual from the function department;

2) Generate the individual data based on some census survey about the whole group;

3) Generate the individual data base on special hypothesis and common experience.

Artificial database contains the artificial population, environment entities and other static date. Geographic data is the core resource of artificial society, which is the basic element to produce virtual environment and artificial population. Environment entities can be generated based on the geographic data, such as government, hospitals and household. Individuals can be put into special environment entities and play special roles in the artificial society.

Artificial population is the basic of the artificial society, which depends on the demographic data, social relation data and so on. In the process of generate the population databases, we can get some demographic data including gender distribution, occupation distribution and so on. These features describe the characters of the real society, which is useful for generating the artificial population. We can generate each individual according to these distributions by iterative arithmetic. Finally we can get the attribute set of each individual, such as id, gender, age, address and so on.

At the same time, we can establish some social networks of individual, such as family network and the family role of each individual. There are some social network we can't gain from traditional demographic data, such as the friendship network and co-worker network. Base on some reasonable hypothesis, experience and common sense can help us to generate these data, which are also useable for some special experiment. For example, establishing contact network based on special hypothesis can also help us to study the spreading mechanism of epidemic.

Based on the census statistics, we can calculate the approximately total number of each type of environment, including residential buildings, companies, kindergarten, elementary school, middle school, universities, supermarkets, shopping malls, restaurants, parks, community geography leisure, gymnasiums, hospitals, clinics and community health clinics. Environmental geographic distribution describes the environment entities and their distribution in the virtual society. Environmental attribute data is used to describe the statistical properties of various types of environmental entities. Based on the distribution and geographic data, we can generate the environment entities, which contain their location, type, storage and so on.

\section{Reconstruct the Initial Artificial Scenario}

Computational experiment is usually based on initial scenario, which is composed of artificial models and database. The scenario is also an active artificial society in special pre-condition, which provides the basic elements and situation of the computational experiment. The construction of the scenario is composed of two parts, namely artificial society models and the initial basic data, which are usually custom-built for different application. Fig. 2 shows the procedure of artificial scenario generation. In the initial scenario, all entities including individual and physical environment are running on the parallel engines based on schedules or some special rules. Models are usually driven by initial basic data, which can be assembled flexibly.

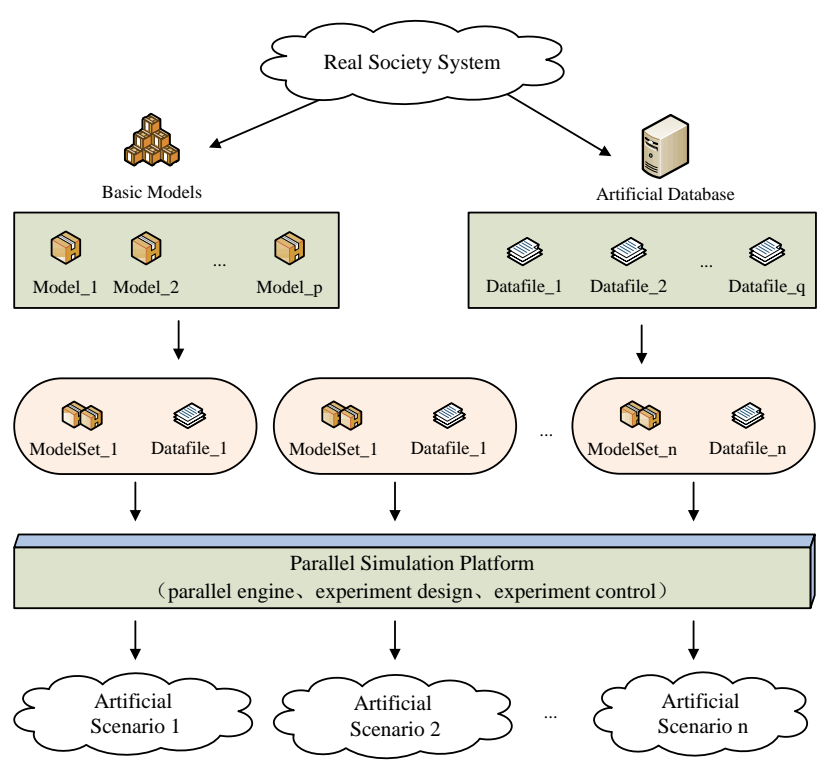

Fig. 2. The process of artificial scenario generation.

For large-scale individual based simulation, the database is always very huge which limit the loading speed. Generating custom-built data file of artificial population can improve the speed. We should choose the appropriate size and attribute parameter of individuals from the database. For example, we may select 1000 student individual with the attributes of health states to simulate the spreading of epidemic in campus. The individual models must match the initial data files, otherwise they can't work synchronically on the parallel engine. The basic database can generate different initial data files, and the instance data is just a subset of the whole database. We can construct different initial scenario base on the same model system and basic database. Computational 
experiment will depend on the initial file not the database, which can improve the efficiency and flexible of simulation.

\section{COMPUTATIONAL EXPERIMENT BASED ON SPECIAL SCENE}

\section{A. The Computational Platform of KD-ACP}

In order to study the scenario-response problems in emergency management, it's necessary to design a platform of artificial social modeling and experimental platform. Base on the ACP theory, national university of defense technology develops the emergency management platform of modeling and simulation, namely KD-ACP. Fig. 3 shows the platform and its tool sets, which is composed of three parts: artificial society module, computational experiment module and parallel control module.

1) Artificial society module depicts the initial scenario, which are composed of model development tool, artificial society editor, geospatial and social environment generation tool.

2) Computational experiment module provides the method and tools to do experiment on special artificial society scene, which contain experimental event configuration tool, intervention measure configuration tool, experiment design tool, and experiment management tool.

3) Parallel execution module associates the real world with the artificial society, which contains artificial society situation tool and open source data registration tool.

The platform supports to establish the artificial society and do computational experiments based on the initial scenario. The construction of artificial society is the precondition, and the computational experiment is the primary method of the society study. The platform provides the ability to load emergency events and control measures, which is based on situation-respond theory and can help us solve some complex problems in emergency management.

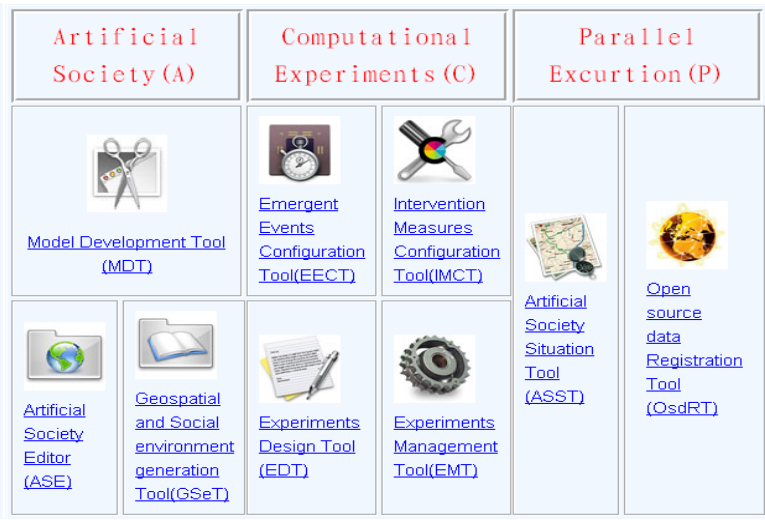

Fig. 3. The computational experiment platform of KD-ACP.

\section{B. The Data Flow in the Simulation}

Fig. 4 depicts the data flow of the platform. In the integrated platform of modeling and simulation, the procedure of the workflow lists as follows:

1) Prepare the basic models and generate the initial data based on statistical data. Attention, the simulation model and initial data must be matched.

2) Build the initial artificial scenario and keep all entities running on the parallel engine. The individual has its own schedule and can active in special social network.

3) Do computational experiment on the virtual society. We can change the operation state of artificial society by loading the emergency events and control measures.

4) Collect and analyze the simulation data. Comparing the simulation data with the real world, we can analyze the behaviors of each individual and evaluate the control measure.

5) Parallel execute the real system and virtual system. We can foster the virtual society and adjust the individual behavior by injecting the dynamic data into the artificial society, which gained from the real society.

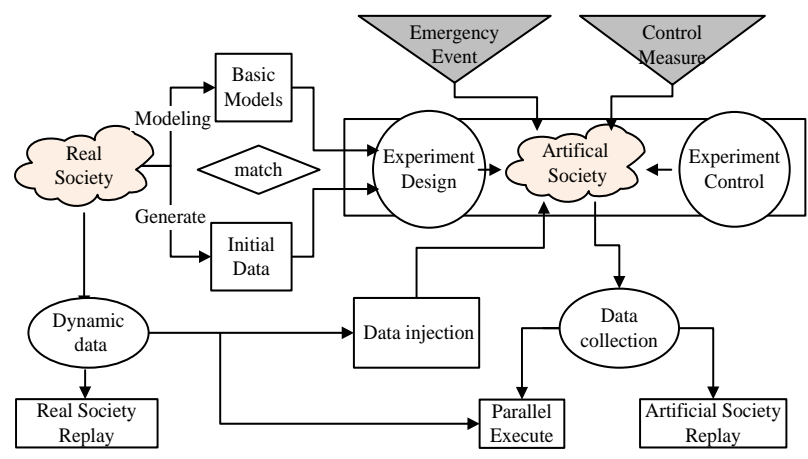

Fig. 4. The workflow of the KD-ACP platform.

In the simulation process, message passing is implemented with MPI. For large-scale individual simulation, the messages and processes are heavyweight, which may affect the performance of the system. We introduce the grid to decrease the correspondence between each individual. The grid is a virtual environment containing individuals and physical environment entities, which is divided by the longitude and latitude. So the message is passed among grids and each individual can gain the message from the grid, which can improve efficiency of simulation.

\section{CASE STUdy: H1NI INFLUENZA}

The experiment is about the simulation of H1N1 influenza emergency in Beijing. H1N1 influenza transmits through close contacts among individuals, such as cough or talking between infectious individual and susceptible individual from close range [13]. We use the basis SIR (Susceptible, Infectious and Recovered) model to present the life cycle of H1N1 influenza disease.

We utilize the census state to reconstruct a virtual city of Beijing, which contains about 19.6 million people and 80 physical environment entities. Population types includes student, worker, teacher, doctor and so on. Environment type includes classroom, workplace, hospital and so on. Each individual can active in special environment according to the schedule. We can establish the social network of each individual, which is also the contact network of epidemic spreading. All entities are running on the parallel engine, which contains 4 computing nodes and 1database node. Each node has 12 cores and 64 GB physical memory. It will take 
1.5 hours to simulate the spreading and intervention process in 60 days.

The event model of H1N1 influenza is composed of diseases modules, infectious resource modules and spreading modules. Fig. 5 shows the template of H1N1 influenza. In the diseases modules, parameters such as disease id, disease name, disease type, infectious rate and death rate are included; In the infectious source model, parameters such as the time and place of disease outbreak, initial disease scale and initial infectious crowds are included; in the spreading modules, parameters such as the contact frequency of each types of environment, the infection rate and the spreading style are included.

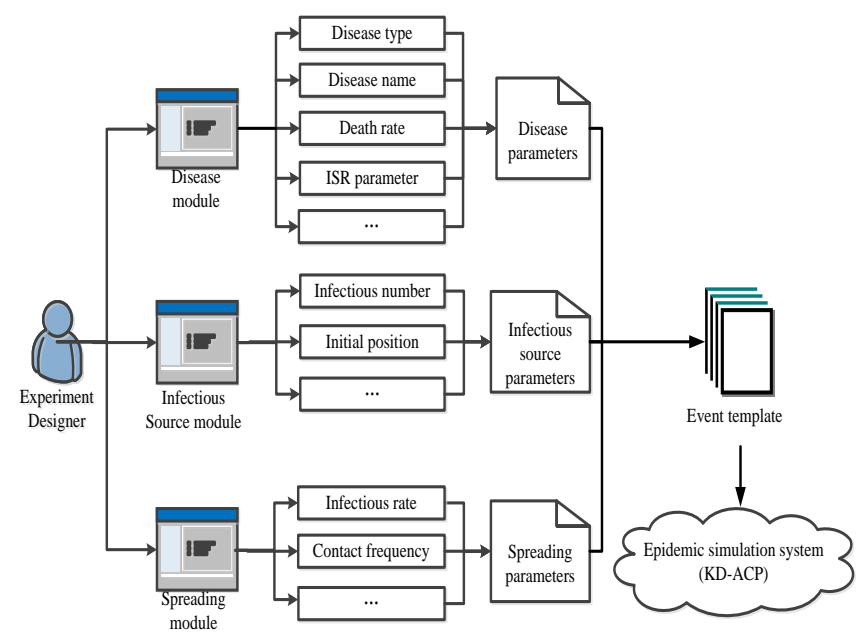

Fig. 5. The event template of H1N1 influenza.

Control measures model includes drug intervention and non-drug intervention measures. Drug intervention module includes the injection of vaccine intervention, hospitals therapy and so on. Non-drug intervention module includes wearing masks, closing public spaces and patient isolation.

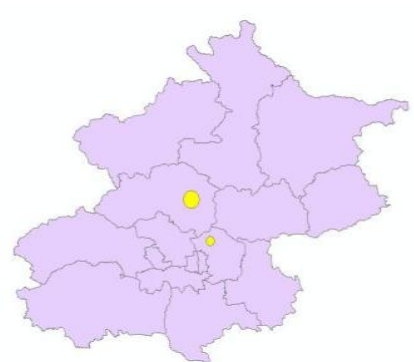

(a)

Fig. 6. The infectious scene of H1NI influenza.

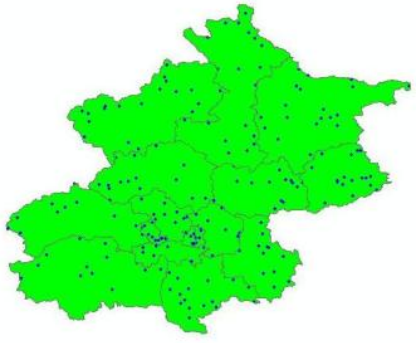

(b)
First, we reconstruct the initial scenario of artificial Beijing, including the corresponding population data and the basic models. Second, we load the event model of H1NI influenza and observe the spreading trend of the disease. Then we load the measures model to control the epidemic disease. Finally, we can do the computational experiments several times by changing the parameters of models, and collect data to analyze the effectiveness of control measures.

In this case, we set the initial infected people is 2 , and the infectious rate is 0.05 . Control measures will be added after 10 days, which includes hospitals therapy, closing public spaces and patient isolation. Finally all individuals have been recovered after 35 day. Fig. 6(a) shows the initial scene of
H1NI influenza, and Fig. 6(b) shows the infectious scene after 10 days. The details of parameter and analysis will not be discussed in this paper.

\section{CONCLUSION}

This paper studies the construction of the artificial society, and provides the method of scenario generation. We establish the KD-ACP platform of computational experiment based on special artificial society scene. In the end, we give a case and study the spreading of H1N1 influenza on the platform. It's unavoidable that there are some aspects also need to improve. Model should be built base on special standard, and more basic data are also needed in the procedure of scenario generation. In the context of emergency management, the event models and control measure model also need more knowledge about special domain.

\section{REFERENCES}

[1] J. A. Campbell, "The SARS commission interim report: SARS and public health in ontario," Biosecurity and Bioterrorism: Biodefense Strategy, Practice and Science, vol. 2, no. 2, pp. 118-126, 2004.

[2] F. Wang, "Artificial societies, computational experiments, and parallel Systems: a discussion on computational theory of complex social economic systems," Complex Systems and Complexity Science, vol. 1, no. 4, pp. 25-35, 2004

[3] J. M. Epstein, "Modelling to contain pandemics," Nature, vol. 460, no.7256, pp. 687-687, 2009.

[4] Z. Fan, W. Duan, B. Chen, and Y. Ge, "Stu dy on the method of multi-agent generation algorithm within special artificial society scene," in Proc.International Conference on Control, UKACC, pp. 1076-1081, 2012.

[5] C. M. Macal and M. J. North, "Agent-based modeling and simulation," in Proc. Winter Simulation Conference, pp. 86-98, 2009.

[6] K. M. Carley, D. B. Fridsma, and E. Casman, "BioWar: scalable agent based model of bioattacks," IEEE Transactions on Systems, Man and Cybernetics, Part A: Systems and Humans, vol. 36, no. 2, pp. 252-265, 2006.

[7] S. S. Fatima, M. Wooldridge, and N. R. Jennings, "An agenda-based framework for multi-issue negotiation," Artificial Intelligence, vol. 152, no. 1, pp.1-45, 2004.

[8] E. Bonabeau, "Agent-based modeling: Methods and techniques for simulating human systems," The National Academy of Sciences of the United States of America, vol. 99, no. 3, pp. 7280-7287, 2002.

[9] F. Wang, "Parallel control and management for intelligent transportation systems: concepts, architectures, and applications," IEEE Transactions on Intelligent Transportation Systems, vol. 11, no. 3, pp. 630-638, 2010.

[10] M. Szell, R. Lambiotte, and S. Thurner, "Multi relational organization of large-scale social networks in an online world," The National Academy of Sciences, vol. 107, no. 31, pp. 13636-13641, 2010.

[11] D. Freeman, "The missing link: China climate change and national security," BICCS Asia, May 2010.

[12] C. Dong, W. Wang, and P. Yang, "DL-based the knowledge description of emergency plan systems and its application," in Proc. Third International Conference on Multimedia and Ubiquitous Engineering, MUE, pp.364-368, 2009.

[13] W. Duan, Z. Cao, Y. Wang, B. Zhu, and D. Zeng, "An ACP approach to public health emergency management: using a campus outbreak of H1N1 influenza as a case study," IEEE Transactions on Systems Man and Cybernetics: Systems, vol. 43, no. 5, pp. 1028-1041, 2013.

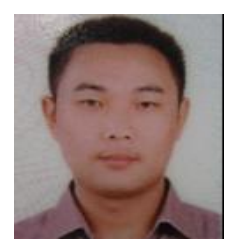

Zhang Peng is a PhD student of college of information system and management, national University of Defense Technology, Changsha, Hunan P. R. China. His is currently research focus on knowledge representation, meta-modeling and model transferring. Now he joins in a national nature science foundation about emergency management, and takes charge of the integrated work about the whole

platform. 
Fan Zongchen is a $\mathrm{PhD}$ student of National University of Defense Technology, Changsha, Hunan P. R. China. His current research is focused on the modeling of public security.

Chen Bin is a lector of National University of Defense Technology, Changsha, Hunan P. R. China. His current research is focused on modeling and simulation about complex system.
Meng Rongqing is a PhD student of National University of Defense Technology, Changsha, Hunan P. R. China. His current research is focused on the computational experiment.

Qiu Xiaogang is a professor of National University of Defense Technology, Changsha, Hunan P. R. China. His current research is focused on artificial society and computational experiment. 